

Hi gh- repet i ti on- $r$ at e opt i cal del ay I i ne usi ng a micromirror array and gal vanomet er mirror for a terahertz system

\begin{tabular}{|l|l|}
\hline 著者 & $\begin{array}{l}\text { K TAHARA H deaki , TAN Nasahi ko, HANGYO } \\
\text { Nasanor } \mathrm{i}\end{array}$ \\
\hline $\begin{array}{l}\mathrm{j} \text { our nal or } \\
\text { publ i cat } \mathrm{i} \text { on } \mathrm{t} \text { i t l e }\end{array}$ & Revi ew of Sci ent i f i c I nst r ument s \\
\hline vol une & 80 \\
\hline number & 7 \\
\hline page $\mathrm{r}$ ange & 076104 1-076104 3 \\
\hline year & $2009-07$ \\
\hline URL & ht t p: //hdl . handl e. net /10098/2109 \\
\hline
\end{tabular}




\title{
High-repetition-rate optical delay line using a micromirror array and galvanometer mirror for a terahertz system
}

\author{
Hideaki Kitahara, ${ }^{\text {a) }}$ Masahiko Tani, and Masanori Hangyo \\ Institute of Laser Engineering, Osaka University, 2-6 Yamadaoka, Suita, Osaka 565-0871, Japan
}

(Received 3 April 2009; accepted 15 June 2009; published online 13 July 2009)

\begin{abstract}
We developed a high-repetition-rate optical delay line based on a micromirror array and galvanometer mirror for terahertz time-domain spectroscopy. The micromirror array is fabricated by using the x-ray lithographic technology. The measurement of terahertz time-domain waveforms with the new optical delay line is demonstrated successfully up to $25 \mathrm{~Hz}$. (C) 2009 American Institute of Physics. [DOI: 10.1063/1.3170578]
\end{abstract}

There is increasing demand for high speed terahertz measurement systems suitable for use in industrial applications, such as the noninvasive inspection of products during mass production. Asynchronous optical sampling (ASOPS) is currently the fastest way to acquire the time waveform in terahertz time-domain spectroscopic (THz-TDS) systems. ${ }^{1,2}$ THz-TDS systems using ASOPS can achieve a scan rate of $10 \mathrm{kHz}$. The frequency resolution of ASOPS is very high, since a whole time period equal to the interval of the sampling laser pulses (usually several tens of nanoseconds) is scanned. However, the system requires a pair of femtosecond lasers whose repetition rates are slightly different and accurately controlled, resulting in high system cost and complexity. In addition, the timing jitter is non-negligible and could be a problem for sensitive measurements. On the other hand, the mechanical optical-delay lines (MODLs) are relatively simple and cost effective. They have good enough performance for low-frequency resolution measurements, except for the measurement of the scan rate, which is limited by the inertia of movable components.

The scan rate of MODL has been improved in various ways in the fields of optical coherence tomography (OCT) and interferometry. ${ }^{3-5}$ In OCT or interferometry, continuous waves and dispersive optical components are usually used. On the other hand, in THz-TDS systems, femtosecond pulses are used, and dispersive optical elements should be avoided. The delay-time span required for THz-TDS is longer than that for OCT, since the wavelength in the terahertz region is longer than the near-infrared region used in OCT. Therefore, high-repetition-rate MODLs optimized for THz-TDS are required.

Recently, delay lines with rotational movement were developed. For example, MODLs with a circular involute mirror, ${ }^{6}$ cylindrical helicoids mirror, ${ }^{7}$ and blade shaped convex mirrors ${ }^{8}$ were reported.

In performing a high speed scan, the scanning mirror should be as simple, small, and light as possible in order to operate the system under a low vibration and noise condi-

\footnotetext{
${ }^{a}$ Present address: Science and Technology Center for Atoms, Molecules, and Ions Control, Plasma Physics Laboratory, Osaka University, 2-1 Yamadaoka, Suita, Osaka 565-0871, Japan. Electronic mail: kitahara@ppl.eng.osaka-u.ac.jp.
}

tion. The galvanometer mirror scanner is a good choice for these purposes. Actually, the MODL with circular involute mirror $^{6}$ uses a galvanometer mirror scanner. Some types of galvanometer mirrors are compact in size and have small torque, which makes them capable of responding up to several hundred hertz. A repetition rate up to $10 \mathrm{kHz}$, which is almost equal to the repetition rate of ASOPS, is achievable by using a resonant galvanometer mirror.

In this paper, we report a high-repetition-rate MODL using a galvanometer mirror and micromirror array for the THz-TDS system. A mirror array consisting of 501 micromirrors was fabricated using $\mathrm{x}$-ray lithography to realize the required accuracy. The MODL was installed into a standard THz-TDS system for the high-repetition-rate measurements of the THz time-domain waveforms.

Figure 1 shows a diagram of the proposed highrepetition-rate ODL. After being reflected from the galvanometer mirror, the laser beam is incident on and reflected back from the aligned micromirrors. If the micromirrors, which reflect the optical beam to the scanning mirror, are aligned on an arclike line and have different distances from the scanning mirror, the delay time can be changed by changing the rotation angle of the scanning mirror. The micromirror size was designed to match to the sizes of the beam diameter on the micromirror. The femtosecond laser used in the THz-TDS has a center wavelength $(\lambda)$ of around $800 \mathrm{~nm}$. A micromirror width $150 \mu \mathrm{m}$ was chosen so that the micromirror would accept a beam with a spot size of $\sim 100 \mu \mathrm{m}$.

We used an x-ray photolithography system of the New SUBARU synchrotron radiation facility in SPring-8 (Super Photon ring-8) for processing the acrylic resin plate to fabricate the micromirror array. ${ }^{9,10}$ It is possible to achieve an accuracy of $50 \mathrm{~nm}$ by $\mathrm{x}$-ray lithography. The polymer microstructure of the lithographie, galvanoformung, and abformung process (LIGA process) was used as the substrate of the micromirror array in this study. The LIGA process has been described in detail previously. ${ }^{9,10}$ The structure of the micromirror array is shown in Fig. 2. The micromirrors are aligned on an arclike line with their distance between the scan mirror and each respective micromirror being increased by $20 \mu \mathrm{m}$ in comparison with the adjacent micromirror. The surface of each micromirror is perpendicular to the lines passing through the center of the galvanometer mirror and 


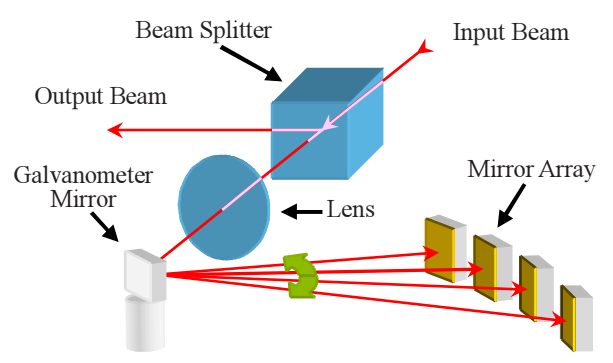

FIG. 1. (Color online) Diagram of the high-repetition rate optical delay line.

each mirror. The center coordinates of the micromirrors are provided by the following equations:

$$
\begin{aligned}
& x=\left(d_{m}-\frac{d_{\text {delay }} \theta}{2 \theta_{\text {max }}}\right) \cos , \\
& y=\left(d_{m}-\frac{d_{\text {delay }} \theta}{2 \theta_{\max }}\right) \sin ,
\end{aligned}
$$

where $d_{\text {delay }}$ is the maximum delay distance, $\theta_{\max }$ is the maximum scan angle of the beam, and $d_{m}$ is the distance of the farthest micromirror from the origin. Here, the center of the scanning mirror is chosen as the origin of the coordinate. In our mirror design, $d_{\text {delay }}, \theta_{\max }$, and $d_{m}$ were $20 \mathrm{~mm}, 60^{\circ}$, and $75 \mathrm{~mm}$, respectively. $\theta_{\max }$ and $d_{m}$ were chosen so that the width of the micromirror was $150 \mu \mathrm{m}$ for the 501 micromirrors with a $20 \mu \mathrm{m}$ distance increment. Since the acrylic resin is transparent to light, the surface of the array was finally coated with a gold thin film. All of the manufacturing processes of the micromirror array were done by Nanocreate Co., Ltd. The photograph of the fabricated mirror array is shown in Fig. 3(a). The roughness of the micromirror surfaces was confirmed to be less than $200 \mathrm{~nm}$ from the scanning electron micrograph (SEM) image shown in Fig. 3(b).

As shown in Fig. 1, a nonpolarized beam splitter was used to introduce the beam reflected from the micromirror array to the detector (a photoconductive antenna). A planoconvex lens with a $200 \mathrm{~mm}$ focal length was used to focus the beam onto the mirror array. The focal length was chosen so that half of the delay length did not exceed the focal depth of the lens $(10.16 \mathrm{~mm})$. Even though a focusing lens with a long focal length was used, the detection efficiency changed with the rotation of the galvanometer mirror due to the change in the beam divergence. This dependence was removed by data processing based on the system calibration. The micromirror array was mounted on a six-axis stage in order to adjust the mirror surfaces precisely for the incident

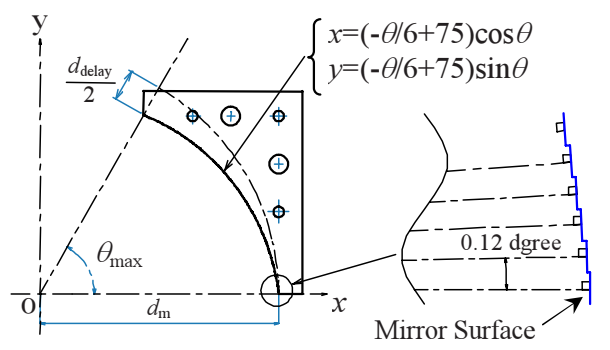

FIG. 2. (Color online) Schematics of the mirror array. The equations provide the center coordinates of the micromirrors. The width of each mirror is approximately $150 \mu \mathrm{m}$. The delay distance $d_{\text {delay }}$ is $20 \mathrm{~mm}$, corresponding to a delay time of $66.7 \mathrm{ps}$. The size of the mirror array is $50 \times 64 \mathrm{~mm}^{2}$.



FIG. 3. (Color online) (a) Photograph of the overall view of the fabricated mirror array and (b) a SEM of the mirror array inside the circle.

beam. The galvanometer mirror (VM500plus; $5 \times 5 \mathrm{~mm}^{2}$ in area, silver coated; GSI Group Inc.) was driven by a triangular waveform voltage supplied by a computer. Figure 4 shows a block diagram of the THz-TDS system used for testing. The photoconductive antenna used as the emitter was biased by the alternating voltage and the terahertz signal was lock-in detected. In addition to the high-repetition-rate optical delay line, an optical delay line with a stepping motor is used for coarse adjustment of the time delay and for the reference measurement for the terahertz waveforms.

When the input laser power was $20 \mathrm{~mW}$, the output laser power after reflection by the beam splitter was $1 \mathrm{~mW}$. The micromirror surface itself had a $72 \%$ reflection coefficient. However, the active efficiency of the micromirror array was reduced to $24 \%$. The low efficiency is attributed to the diffraction at the edges of micromirror and the inaccuracy of the mirror surface alignment near the edges. The transmission loss of the planoconvex lens was $17 \%$ and the nonpolarized beam splitter gave a transmission loss of $75 \%$ for the round trip of the laser pulse. Therefore, the total transmission efficiency was reduced to $5 \%$ for the present system. The scan rate of the galvanometer mirror can be operated up to $25 \mathrm{~Hz}$ and is limited by the numerical calculation speed of the computer in the present system.

Figure 5 shows a magnification of the waveform at the main peak, which consists of many smaller peaks. This waveform was obtained by accumulating 128 scans at a scan rate of $1 \mathrm{~Hz}$. The small peaks are caused by the fluctuation of the probe beam intensity: the intensity of the probe beam at the detector decreases when the probe beam strikes the boundary of two adjacent micromirrors because of the diffraction. The fluctuation component is removed by smooth-

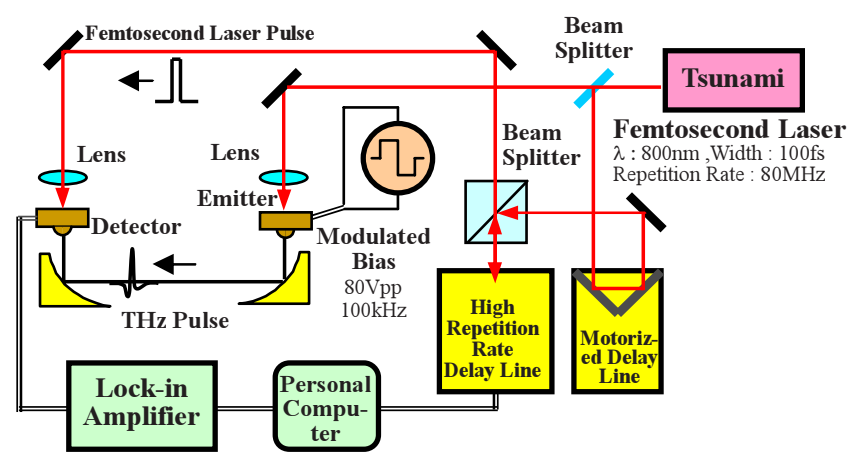

FIG. 4. (Color online) Diagram of the THz-TDS system for the highrepetition-rate optical delay line. 


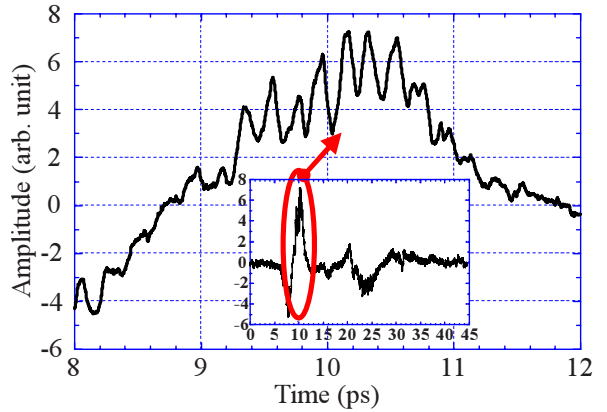

FIG. 5. (Color online) Shape of the main peak with an accumulation of 128 scans at a scan rate of $1 \mathrm{~Hz}$. The inset shows the whole waveform of the terahertz pulse.

ing at the cost of the time resolution. Figure 6(a) shows the waveform obtained with a single scan at a scan rate of $25 \mathrm{~Hz}$. Since the waveform contains a large amount of noise and it was difficult to find the main peak, it was smoothed in order
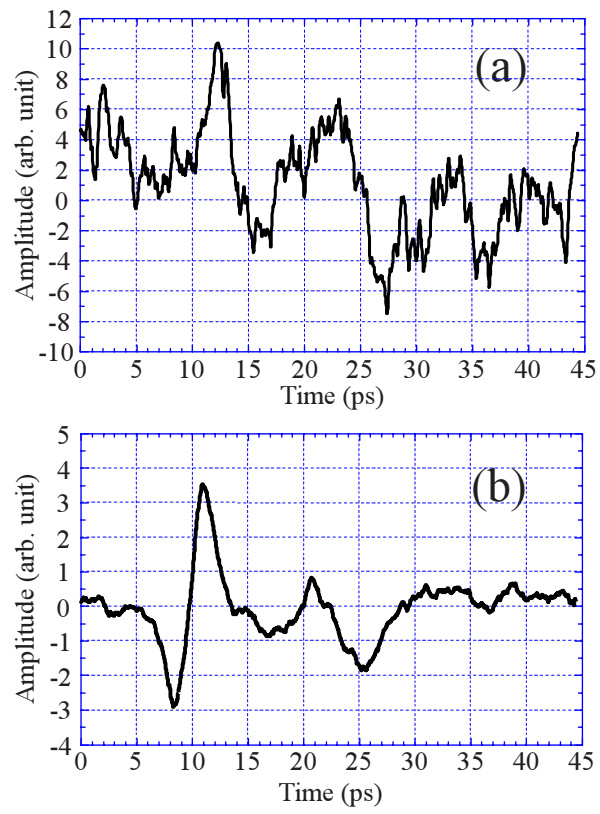

FIG. 6. (Color online) Waveform of the terahertz pulse measured with the high-repetition-rate optical delay line. The waveforms obtained by (a) a single scan and (b) an average of 128 scans at a scan rate of $25 \mathrm{~Hz}$ are shown. to eliminate the short-period fluctuation seen in Fig. 5. Figure 6(b) shows the integrated waveform obtained by the accumulation of 128 scans with smoothing. In the smoothed waveform, the signal-to-noise ratio is improved and the main peak is recognized clearly.

In conclusion, we demonstrated a high-repetition-rate optical delay line consisting of a galvanometer mirror and micromirror array. The mirror array was fabricated using $\mathrm{x}$-ray lithography. The waveform of the terahertz wave was obtained successfully at a scan rate of $25 \mathrm{~Hz}$. Compared to the high-repetition-rate optical delay lines reported so far, ${ }^{6-8}$ the present system has the advantage that the delay time can be changed quickly for any series desired by applying the voltage waveform to the galvanometer mirror. For example, a differential terahertz image between time delays $t_{1}$ and $t_{2}$ can be obtained without scanning the whole waveforms of terahertz pulses in a short time. As for the scan rate, up to $300 \mathrm{~Hz}$ can be achieved using a high speed computer. We are planning to improve the structure and fabrication process of the micromirror array to increase the reflectance of the mirror array and time resolution.

This work was supported partly by the Terahertz Optics Project for Medical Application led by Professor Jun-ichi Nishizawa, organized by the Ministry of Education, Culture, Sports, Science and Technology of Japan. The authors thank Kenji Yamashita and Takashi Inoue for their contribution in preparing the micromirror array.

${ }^{1}$ D. W. van der Weide, J. Murakowski, and F. Keilmann, IEEE Trans. Microwave Theory Tech. 48, 740 (2000).

${ }^{2}$ T. Yasui, E. Saneyoshi, and T. Araki, Appl. Phys. Lett. 87, 061101 (2005).

${ }^{3}$ A. M. Rollins and Jo. A. Izatt, Handbook of Optical Coherence Tomography, edited by B. E. Bouma and G. J. Tearney (Marcel Dekker, New York, 2002), Chap. 4, p. 99.

${ }^{4}$ M. Lai, Appl. Opt. 40, 6334 (2001).

${ }^{5}$ W. Y. Oh, S. H. Yun, G. J. Tearney, and B. E. Bouma, Opt. Lett. 30, 3159 (2005).

${ }^{6}$ J. Xu and X.-C. Zhang, Opt. Lett. 29, 2082 (2004).

${ }^{7}$ J. Xu, Z. Lu, and X.-C. Zhang, Electron. Lett. 40, 1218 (2004).

${ }^{8}$ G. J. Kim, S. G. Jeon, J. I. Kim, and Y. S. Jin, Jpn. J. Appl. Phys., Part 1 46, 7332 (2007).

${ }^{9}$ E. W. Becker, W. Ehrfeld, A. Maner, and D. Munchmeyer, Microelectron. Eng. 4, 35 (1986).

${ }^{10}$ Y. Utsumi, T. Kishimoto, T. Hattori, and H. Hara, Jpn. J. Appl. Phys., Part 144,5500 (2005). 